\title{
Skin and Proximity Effects in Electrodes and Furnace Shells
}

\author{
EGIL VÅLANDSMYR HERLAND, MANUEL SPARTA (D, \\ and SVENN ANTON HALVORSEN (D)
}

\begin{abstract}
A review of two-dimensional (2D) analytical models of skin and proximity effects in large industrial furnaces with three electrodes arranged in an equilateral triangle is given. The models cover three different cases: one electrode only, three electrodes where two are approximated by line currents, and induced shell currents where all electrodes are approximated by line currents. The first two models show how the skin and proximity effects depend on electrode material properties and size, and the distance between the electrodes. The third model shows how the strength of the induced shell currents will depend on electrode position and furnace size. These models are compared to numerical studies including distributed electrodes and shell currents. The analytical models are accurate when induced shell currents can be disregarded. However, strong shell currents may have a significant impact on the current distribution within the electrodes. This electrode-shell proximity effect competes with the electrode-electrode proximity effect. Finally, the 2D models have been compared with three-dimensional (3D) case studies of large industrial furnaces. In $3 \mathrm{D}$, the shell currents are significantly smaller than what are predicted by the $2 \mathrm{D}$ models, but they are sufficiently strong to cause a significant correction of the electrode current density.
\end{abstract}

https://doi.org/10.1007/s11663-019-01651-8

(C) The Author(s) 2019

\section{INTRODUCTION}

IN many metal-producing units, the energy needed for the primary reactions are delivered by electric currents through large electrodes. Examples of such units are slag resistance furnaces and electric arc furnaces, used in the production of steel, ferroalloys, calcium carbide and silicon. ${ }^{[1,2]}$ Typically, three-phase alternating current (AC) circuits using three or more electrodes are used, operating at the grid frequency of 50 or $60 \mathrm{~Hz}$. Correct and stable operation of the electrodes is of crucial importance for successful and cost-effective operation of the furnace. ${ }^{[3,4]}$ Detailed understanding of current densities, thermal conditions and mechanical stresses is needed to address electrode problems such as electrode breakages and electrode consumption. ${ }^{[5-7]}$ Also, for designing the furnace and for understanding its process, the current-carrying capabilities and sizing of the electrode play an important role. ${ }^{[8-10]}$

EGIL VÅLANDSMYR HERLAND is with NORCE Norwegian Research Centre AS, Universitetsveien 19, 4630 Kristiansand, Norway and also now with Elkem ASA Technology, Fiskåveien 100, 4621 Kristiansand, Norway. MANUEL SPARTA and SVENN ANTON HALVORSEN are with NORCE Norwegian Research Centre AS. Contact e-mail: svha@norceresearch.no

Manuscript submitted January 28, 2019.

Article published online August 26, 2019.
The electrodes are made of electrically conductive graphite or baked carbon material, and their main function is to carry the electric currents needed for powering the chemical reactions of the process. Although direct current (DC) furnaces can be found in the industry, most units use AC current, and in AC electrodes, there is considerable concentration of currents close to the surface; the well-known skin effect. ${ }^{[2]}$ The skin effect is caused by eddy currents that are induced by the changing magnetic field produced by the current. The current density distribution in an electrode with skin effect is found by solving Maxwell's equations, and for the typical case with vertical currents and cylinder geometry, the current density is axisymmetric and given in terms of Bessel functions. ${ }^{[11,12]}$ In addition to the skin effect, there are also considerable proximity effects caused by eddy currents opposing the magnetic field produced by the currents in the neighboring electrodes. The proximity effects typically yield an asymmetric current density in the electrodes. ${ }^{[13-18]}$ Although these effects are significant and wellknown, ${ }^{[19-23]}$ they are often neglected in electrode models which typically concentrate on a single electrode. ${ }^{[7,24-26]}$

Most industrial furnaces have a furnace shell. Its function is to carry the weight of the reactants, products and lining materials in the furnace, and it is typically made of structural steel. However, such materials are 
both highly conductive and ferromagnetic, and strong eddy currents are induced in the furnace shell. A theoretical and experimental study of this phenomenon, can be found in Reference 27. When present, these shell currents may also modify the currents of the electrode, effectively creating electrode-shell proximity effects.

In this work, mathematical models of skin and proximity effects in three-phase AC furnaces with the typical triangular electrode arrangement are studied. Analytical models of skin and proximity effects found in the literature are reviewed and supplemented with numerical simulations of more representative 2D geometries. In particular, the electrode-shell proximity effects are included in this study. The strength of the skin and proximity effects mainly depend upon electrical material properties and important geometrical sizes such as the furnace diameter, electrode diameter and electrode-electrode distance. The main goal is to study how these electromagnetic effects depend on the geometrical sizes. Also, both the analytical models and the 2D numerical models are compared with numerical simulations of industrial furnaces in 3D.

In Section II, the basics of the models are reviewed. The numerical models are described in Section III. Section IV presents the numerical results including discussions and comparisons with the models of Section II. The conclusions are given in Section V.

\section{REVIEW OF MATHEMATICAL MODELS}

In this section, three analytical models are reviewed. The first model describes the skin effect in a single electrode. The second model describes both skin and proximity effects in electrodes assuming the typical triangular electrode configuration used in three-phase AC furnaces. The third model describes the induced currents in the furnace steel shell.

\section{A. Skin Effect}

A model that describes the skin effect in an electrode can be found by solving Maxwell's equations for the geometry shown in Figure 1. That is, the electrode is assumed to be cylinder-shaped with radius $R$, and the AC current density $\mathbf{J}$ is assumed to be vertical, $\mathbf{J}=J_{z} \hat{\mathbf{z}}$, with total current amplitude $I$. In the derivation, it is assumed that the furnace operates at low AC frequency $f$ and that the electric field $\mathbf{E}$ and the magnetic field $\mathbf{B}$ are time-harmonic. Importantly, it is also assumed that the electric current density obeys Ohm's law,

$$
\mathbf{J}=\sigma \mathbf{E},
$$

where $\sigma$ is the electrical conductivity. More details on the low-frequency Maxwell's equations can be found in Appendix A.1. The solution of these equations in a conductive domain exhibits the skin effect. The current will then flow close to the surface of the conductor. The skin effect is quantified by the skin depth $\delta$, given by

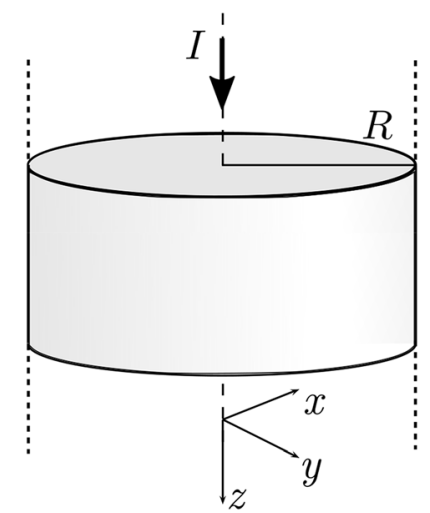

Fig. 1-Model geometry of single electrode.

$$
\delta=\sqrt{\frac{1}{\pi f \mu \sigma}},
$$

where $\mu$ is the magnetic permeability. The skin depth is the penetration depth where the current density is reduced by the factor $1 / e$ from the surface.

The resulting expressions for the geometry of Figure 1 are quoted in Appendix A.2. Most importantly, the expression for the $\mathrm{AC}$ resistance factor $R_{\mathrm{AC}} / R_{\mathrm{DC}}$ is given in Eq. [A7], and the expression for the root-meansquared current density $J_{z, \text { rms }}$ is given in Eq. [A12]. Note that the current density expression is axisymmetric, i.e., the only spatial dependence is with the radial coordinate $r$. Also note that when $J_{z, \mathrm{rms}}$ is normalized by the DC current density $J_{z, \mathrm{DC}}=I_{\mathrm{rms}} /\left(\pi R^{2}\right)$, it only depends on two nondimensional numbers, namely the nondimensional radius $r / R$ and the ratio $R / \delta$, which can be thought of as inverse nondimensional skin depth. In other words, the shape of the current density depends only on $R / \delta$. The strength of the skin effect is controlled by $R / \delta$. When $R / \delta \ll 1$, the skin effect is negligible, and the current density is uniform. When $R / \delta \sim 1$, the current starts to accumulate near the surface of the electrode, and when $R / \delta \gg 1$, the current flows only within the skin of the electrode. The importance of $R / \delta$ is also seen when studying the resistance of the electrode (see Eq. [A7]). The $\mathrm{AC}$ resistance factor $R_{\mathrm{AC}} / R_{\mathrm{DC}}$, which quantifies the increased resistance associated with skin effect in AC electrodes, depends only on $R / \delta$.

\section{B. Electrode-Electrode Proximity Effect}

Analytical expressions for the skin and proximity effects in the electrodes of the three-phase AC furnaces with the typical triangular electrode configuration are available in the literature. ${ }^{[20,21]}$ The system under consideration is sketched in Figure 2. In the furnace, the electrodes are oriented in the vertical direction, so Figure 2 can be regarded as the horizontal cross section of the 3D electrode configuration. Three electrodes with the same radius $R$ are placed in the corners of an 
equilateral triangle, and the center-center distance between the electrodes is $d$. From the perspective of electrode 1 , we define an azimuthal angle $\theta$ oriented such that the leading electrode (2) is found at $\theta=0$, and the trailing electrode (3) at $\theta=\pi / 3$. Note that in the derivation of Reference 20, the currents of the neighboring electrodes are described as line currents. This simplified description is illustrated in the figure. Furthermore, all current densities are assumed to be vertical, $\mathbf{J}_{i}=J_{i, z} \hat{\mathbf{z}}$, where index $i$ denote the electrode. The electrical properties are fixed for all electrodes. The amperage is the same for all electrodes, but there is a 120-degree phase difference between the electrodes. The resulting expressions are quoted in Appendix A.3. In these expressions, it is assumed that electrode 2 is leading electrode 1 .

The most important expressions are the root-meansquared current density in Eq. [A15] and the AC resistance factor in Eq. [A20]. In these expressions, the electrode-electrode proximity effects are included as a series expansion in orders of the nondimensional number $R / d$, which is the ratio between the electrode radius and the electrode-electrode distance. For any practical application, the series converges with few terms, and the formula can be implemented efficiently.

Together with $R / \delta, R / d$ is important for determining the strength of the proximity effects. In the limit when $R / d \rightarrow 0$, Eqs. [A15] and [A20] reduce to the skin effect expressions of Eqs. [A12] and [A7]. With increasing values of $R / d$, the importance of the proximity effect terms increases. Note that these terms also depend on the azimuthal angle $\theta$, and the current density is no

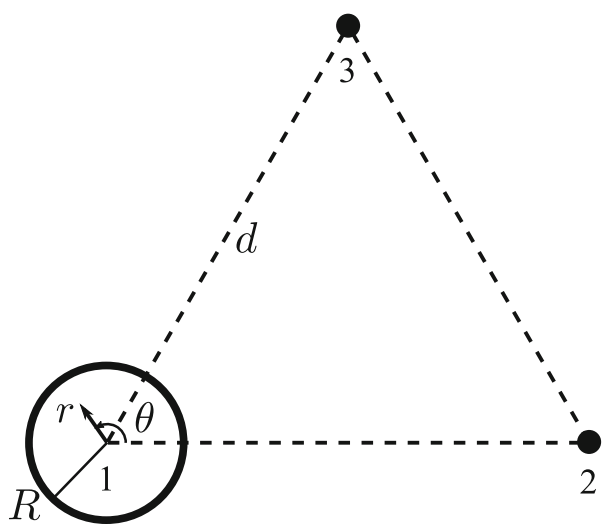

Fig. 2-2D cross section of a system with triangular electrode configuration. longer axisymmetric. The proximity effect yields a shift of the current density towards the leading electrode. ${ }^{[13-18,20,22]}$

The analytical models of Sections II-A and II-B, were applied to the industrial setups summarized in Table I. In Figure 3, current density profiles are calculated using Eqs. [A12] and [A15]. The hallmarks of both the skin and proximity effect can clearly be appreciated. In Figure 4, the time-averaged power density, given by $p_{1}=\left|J_{z, 1}\right|^{2} /(2 \sigma)$, is calculated for the electrode 1 surface using Eq. [A13]. The values have been normalized with the DC power density, $p_{\mathrm{DC}}=$ $J_{z, \mathrm{DC}}^{2} / \sigma$. The figure illustrates both the skin and the electrode-electrode proximity effects. The skin effect is responsible for higher power density in the surface compared with the DC power density, i.e., $p_{1}(R, \theta) / p_{\text {DC }}>1$. The asymmetric form of the curves with accumulation of power close to the leading electrode is caused by the electrode-electrode proximity effect. The asymmetry can also be studied by calculating the ratio

$$
\frac{\Delta p_{R}}{\overline{p_{R}}}=\frac{\max \left[p_{1}(R, \theta)\right]-\min \left[p_{1}(R, \theta)\right]}{\overline{p_{1, R}}}
$$
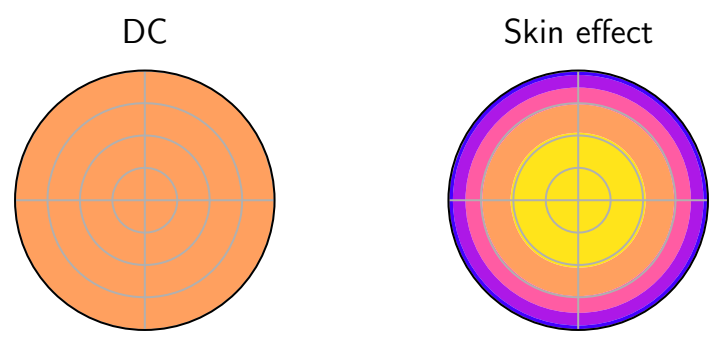

Skin and proximity effect
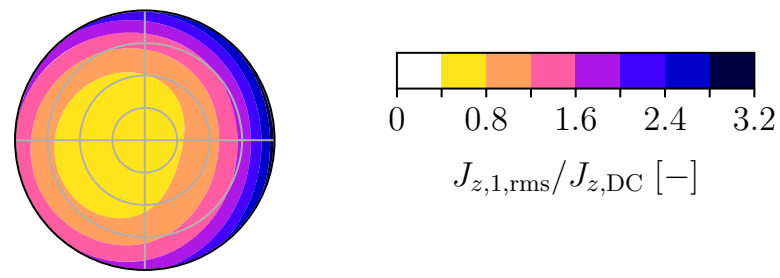

Fig. 3 - Contour plots of current density profiles for an electrode subject to different electromagnetic effects. The values $R / \delta=2.8$ and $R / d=0.23$ used here correspond to case $\mathrm{B}$ of Table $\mathrm{I}$.

Table I. Properties for Some Industrial Three-Phase Furnaces with Søderberg Electrodes

\begin{tabular}{llccccccccc}
\hline ID & \multicolumn{1}{c}{ Process } & $\sigma\left(\mathrm{Sm}^{-1}\right)$ & $R(\mathrm{~m})$ & $d(\mathrm{~m})$ & $R_{\mathrm{f}}(\mathrm{m})$ & $\delta(\mathrm{m})$ & $R / \delta(-)$ & $R / d(-)$ & $d /\left(\sqrt{3} R_{\mathrm{f}}\right)(-)$ & $\mathrm{Reference}$ \\
\hline A & ferromanganese & $5.0 \times 10^{4}$ & 0.95 & 4.9 & 7.0 & 0.32 & 3.0 & 0.19 & 0.40 \\
B & ferrosilicon & $5.0 \times 10^{4}$ & 0.90 & 4.0 & 5.5 & 0.32 & 2.8 & 0.23 & 0.42 \\
C & ferrochrome & $2.8 \times 10^{4}$ & 0.78 & 3.8 & 5.1 & 0.43 & 1.8 & 0.21 & 0.43 \\
\hline
\end{tabular}

The properties are electrical conductivity $\sigma$, electrode radius $R$, electrode-electrode distance $d$, shell radius $R_{\mathrm{f}}$, skin depth $\delta$ and nondimensional numbers. Frequency $f=50 \mathrm{~Hz}$ and vacuum magnetic permeability $\left(\mu=\mu_{0}=4 \pi \times 10^{-7} \mathrm{Hm}^{-1}\right)$ are used in all cases. 


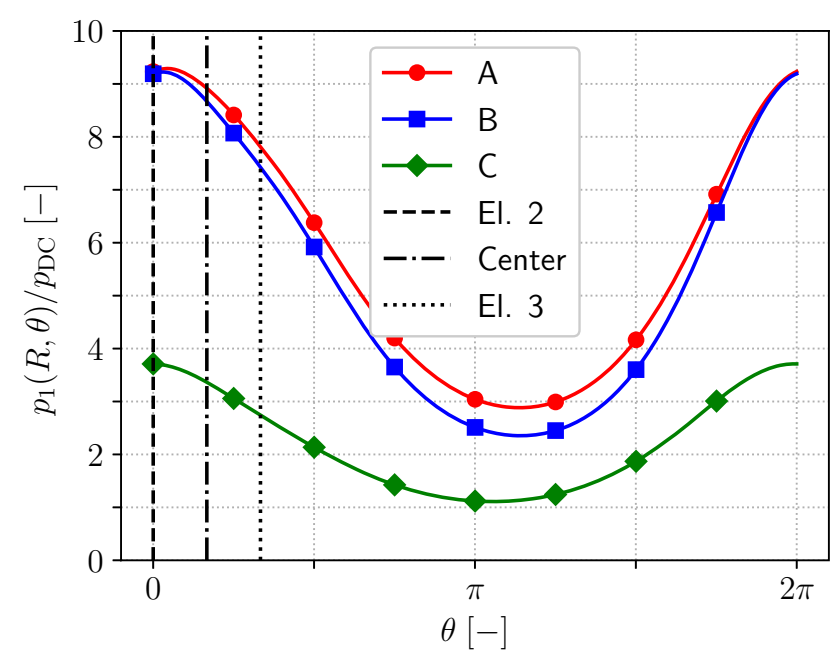

Fig. 4-Normalized power density at electrode surface, as a function of $\theta$, for the cases of Table I. Directions to other electrodes and furnace center are given by vertical lines (see Fig. 2).

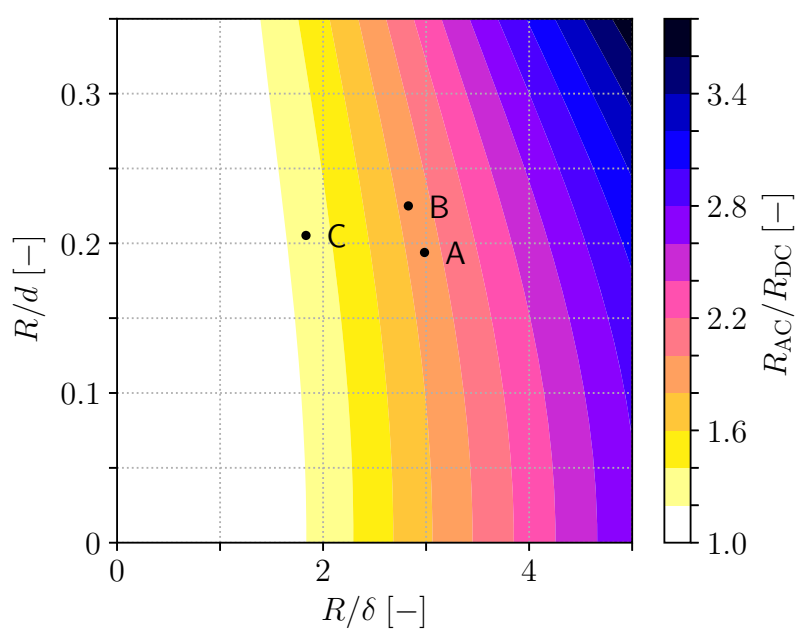

Fig. 5-Contour plot of the $\mathrm{AC}$ resistance factor $R_{\mathrm{AC}} / R_{\mathrm{DC}}$, as a function of nondimensional numbers $R / \delta$ and $R / d$. Labels $\mathrm{A}, \mathrm{B}$ and $\mathrm{C}$ denote the cases of Table I.

where $\max \left[p_{1}(R, \theta)\right]$ and $\min \left[p_{1}(R, \theta)\right]$ denote the maximum and minimum of the surface power density curves (see Figure 4), and $\overline{p_{R}}$ denotes the average value. The result is shown in Supplementary Figure S-1 (refer to electronic supplementary material).

For electrodes with skin and proximity effects, the inhomogeneous current distributions yield higher resistance than for DC electrodes. Figure 5 shows a contour plot of the AC resistance factor $R_{\mathrm{AC}} / R_{\mathrm{DC}}$, calculated by Eq. [A20]. This ratio is a function of the nondimensional numbers $R / \delta$ and $R / d$. The electrode resistance increases with both numbers. It is also possible to study the proximity effect factor $R_{\mathrm{AC}} / R_{\mathrm{AC}, \mathrm{s}}$, where $R_{\mathrm{AC} . \mathrm{s}}$ is the $\mathrm{AC}$ resistance for an electrode with only the skin effect, calculated by Eq. [A7]. This ratio quantifies the
Table II. The AC Resistance Factor and the Proximity Effect Factor for the Example Electrodes of Table I

\begin{tabular}{lcc}
\hline ID & $R_{\mathrm{AC}} / R_{\mathrm{DC}}(-)$ & $R_{\mathrm{AC}} / R_{\mathrm{AC}, \mathrm{s}}(-)$ \\
\hline $\mathrm{A}$ & 1.91 & 1.08 \\
$\mathrm{~B}$ & 1.86 & 1.11 \\
$\mathrm{C}$ & 1.28 & 1.07 \\
\hline
\end{tabular}

additional resistance caused by the electrode-electrode proximity effects. The result is shown in Supplementary Figure S-2. Table II shows values for the AC resistance factor and the proximity effect factor for the electrodes of Table I.

Accurate calculation of the skin and proximity effects in the electrodes have important practical consequences for furnace operation and, in particular, electrode operation. Electrode conditions and operating procedures are optimized using electrode models, in which the calculation of temperatures and thermal stresses in the electrodes are based upon accurate estimates of the Joule heating inside the electrode. ${ }^{[7]}$ As shown here, the models should include proximity effects in order to be sufficiently accurate. Also, $R_{\mathrm{AC}} / R_{\mathrm{DC}}$ goes directly into typical expressions for electrode load factors and the current-carrying capacities of electrodes. ${ }^{[5,8,10]}$ In these expressions, the electrode load factor increases with $R_{\mathrm{AC}} / R_{\mathrm{DC}}$. For example, decreasing proximity effects by increasing the electrode-electrode distance $d$ will increase the current-carrying capacity of an electrode. Consequently, accurate estimates of electrode skin and proximity effects are needed for determining key parameters related to operation and furnace design.

\section{Shell Currents}

Relatively simple expressions for the shell currents induced by the three-phase electrode currents can be found in the literature. ${ }^{[27,29,30]}$ In these works, the electrodes are modelled as wires, and the cylindrical shell is assumed to be so thick that all fields decay within. The latter assumption is good when the shell skin depth is much smaller than its thickness. For typical steel with $\sigma=6 \times 10^{6} \mathrm{Sm}^{-1}$ and $\mu=\mu_{\mathrm{r}} \mu_{0}$, with relative permeability $\mu_{\mathrm{r}}=200$, the skin depth $\delta_{\mathrm{sh}}=2 \mathrm{~mm}$ at $f=$ $50 \mathrm{~Hz}$. Hence, the approximation is good for industrial furnaces with shell thicknesses that typically are an order of magnitude larger than the skin depth.

The configuration is shown in Figure 6, where $R_{\mathrm{f}}$ is the radius of the shell. The configuration and the electrode current amplitudes and phases are the same as described in Section II-B. The current density in the shell was calculated by Reference 30 . Since $\delta_{\mathrm{sh}} \sim 1 \mathrm{~mm}$, the current of the steel shell is well approximated as an effective surface current $K(\varphi)$ only dependent on the azimuthal angle $\varphi$ of the furnace. The resulting expressions are quoted in Appendix A.4. 


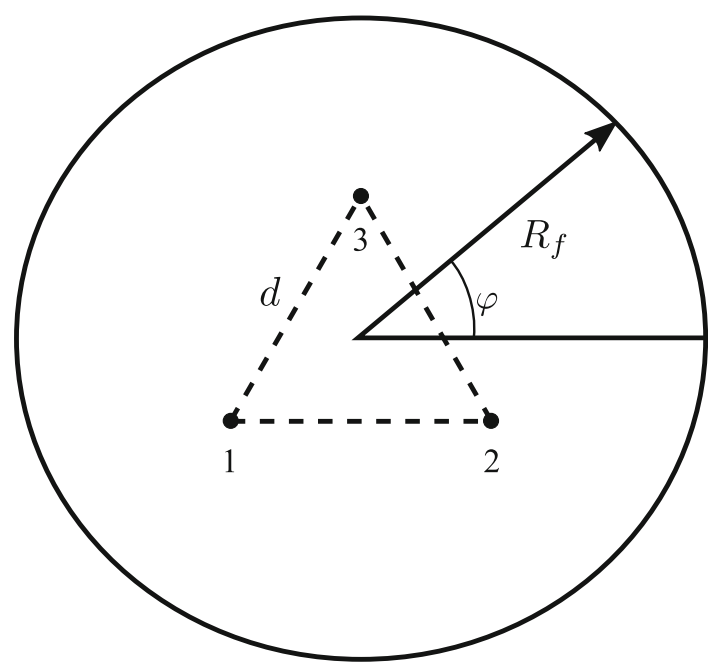

Fig. 6 - Geometry for calculation of shell currents. The numbers identify the different electrodes.

In Eq. [A23] is an expression for the total induced current that can be associated with the shell section nearest electrode 1. This expression reveals that the nondimensional number $d /\left(\sqrt{3} R_{\mathrm{f}}\right)$ is important for determining the strength of the induced shell currents. The distance from the furnace center to the electrode center is equal to $d / \sqrt{3}$, so $d /\left(\sqrt{3} R_{\mathrm{f}}\right)$ is the ratio between the electrode center radius and the shell radius of the furnace. When this number is small, the induced currents of the shell are small. This is reasonable, since this limit corresponds to the situation where the three electrodes are close and the shell is far away. The threephase electrodes can then be considered as a single conductor with zero net current, and consequently, there will not be any induced currents in the shell. In the opposite limit, when $d /\left(\sqrt{3} R_{\mathrm{f}}\right) \rightarrow 1, I_{\mathrm{sh}, 1} \rightarrow-I$. That is, the total current in the shell section is equal to the current of the electrode, but with opposite phase. This corresponds to three separate, non-interacting electrodewall systems. Note that this limit is not realistic in a furnace since it would imply that the electrodes are line currents.

The shell currents can also be studied graphically in Figure 7. The figure shows the normalized root-meansquared current density $K_{\mathrm{rms}}(\varphi) / K_{0}$, calculated using Eq. [A21] and normalized with $K_{0}=I_{\mathrm{rms}} /\left(2 \pi R_{\mathrm{f}}\right)$. Note that $K_{0}$ is equal to the surface current density that one would obtain if the current of one electrode were uniformly distributed throughout the inner surface of the shell. However, since there are three electrodes in the furnace, it can be seen that $K_{\mathrm{rms}}(\varphi) / K_{0}$ on average approaches 3 when electrodes are close to the shell. The peak values are higher, since the induced shell currents are nonuniform and concentrate close to the electrodes. Supplementary Figure S-3 shows the integrated shell current in the electrode 1 section of the shell, $\left|I_{\mathrm{sh}, 1} / I\right|$, as calculated by Eq. [A23].

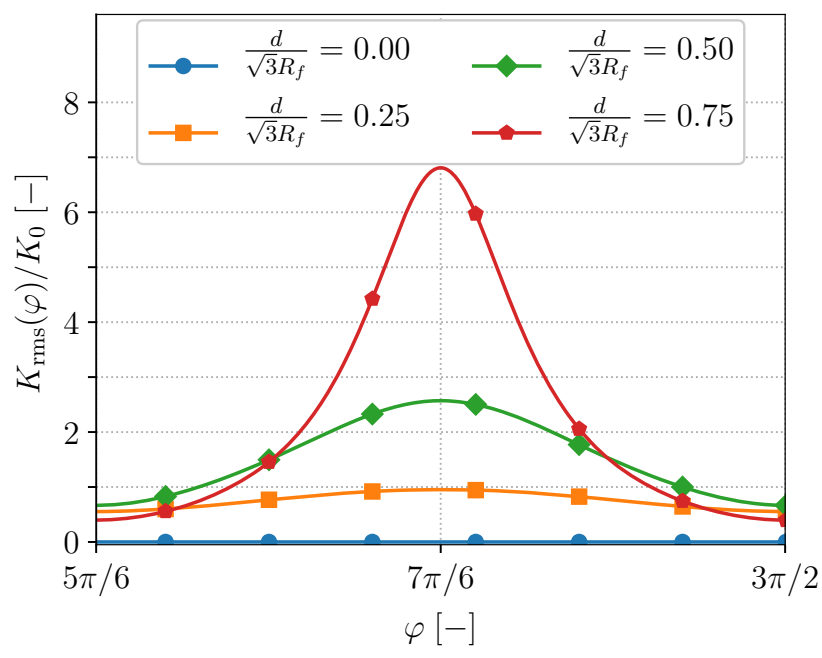

Fig. 7-Normalized shell currents, $K_{\mathrm{rms}}(\varphi) / K_{0}$ as a function of $\varphi$, for different values of $d /\left(\sqrt{3} R_{\mathrm{f}}\right)$. The figure shows results for the electrode 1 section (when $\varphi \in[5 \pi / 6,3 \pi / 2$ ), see Fig. 6). The results are the same for electrodes 2 and 3 .

\section{NUMERICAL MODEL DESCRIPTION}

In deriving the useful expressions of Section II, important assumptions have been made. The spatial extent of neighboring electrodes in the horizontal cross section have been neglected, and the steel shell is assumed to be thick. In the numerical model, we can account for the spatial extent of the electrodes, and for the finite thickness of the shell. Additionally, the numerical model allows for studying the proximity effects between the induced shell currents and the electrodes. The domains of the numerical model are shown in Figure 8.

The numerical calculations in this work were performed by using the Magnetic Field interface in the AC/DC module of the COMSOL Multiphysics software. ${ }^{[31,32]}$ In this interface, Maxwell's equations in Eq. [A2] are solved by a formulation in terms of the magnetic vector potential $\mathbf{A}$. The electromagnetic fields are then given by $\mathbf{B}=\nabla \times \mathbf{A}$ and $\mathbf{E}=$ $-\partial \mathbf{A} / \partial t$. That is, the electric potential is not solved for except within the electrodes where the coil domain feature was used. ${ }^{[32]}$ The equations are discretized using the finiteelement method with quadratic element order. To avoid influence from the boundaries of the simulation domain, the electrodes and furnace shell are surrounded by dielectric material with a radius of $10 R_{\mathrm{f}}$ and with infinite element domains towards the outer boundary of the simulation domain. ${ }^{[31]}$ At the outer boundary, the magnetic insulation boundary condition was used. In most of the simulation domain, triangular mesh elements were used, with high mesh density in the electrodes in order to resolve the gradients caused by the skin and proximity effects. For the thin steel shell with very small skin depth, thin quadrilateral boundary layer mesh elements were used in order to resolve the large gradients close to the surface. In total, there were typically around $1 \times 10^{5}$ mesh elements for each calculation.

In industrial furnaces, the furnace shell is typically made of magnetic steel. In this model, the steel shell has been modelled in a simplified manner, as a linear 


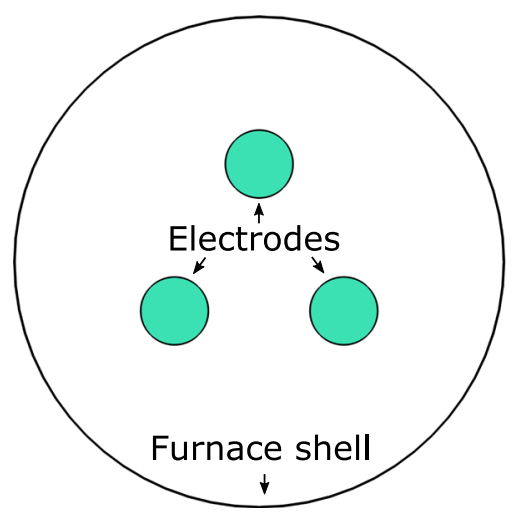

Fig. 8-Domains of the numerical model. The figure shows the electrodes in the triangular configuration inside the thin steel shell.

magnetic material with a constant $\mu_{\mathrm{r}}=200$. Moreover, $f=50 \mathrm{~Hz}, R=1 \mathrm{~m}$ and $I_{\mathrm{rms}}=115 \mathrm{kA}$ and steel shell thickness $t_{\mathrm{sh}}=25 \mathrm{~mm}$. Since the model is relatively simple and the solution typically converges in less than a minute, many calculations were performed in order to map out the parameter space spanned by the nondimensional numbers of the system. This was done by varying parameters $\sigma, d, R_{\mathrm{f}}$ and the steel shell electrical conductivity $\sigma_{\text {sh }}$.

\section{RESULTS AND DISCUSSION}

\section{A. Triangular Electrode Configuration}

We have studied the current and power distribution for the triangular electrode configuration numerically. Compared with the analytical expressions of Section II-B, the numerical model is more accurate since all electrode currents are distributed throughout the electrode cross section. In total, results for 480 different parameter combinations have been calculated numerically. This was done by choosing 20 different values of $\sigma$ and 24 different values of $d$ such that all combinations of $R / \delta \in\{0.25,0.50, \ldots, 5.00\} \quad$ and $R / d \in\{0.015$, $0.030, \ldots, 0.360\}$ were studied.

As an example, the normalized current densities for $R / \delta=3.0$ and $R / d=0.225$ are shown in Figure 9. The ratio between the numerical and analytical (Eq. [A15]) model is given, showing that both models yield similar results. Differences up to $\approx 1.5$ pct can be seen. In Figure 10, the relative power density on the surface of electrode $1, p_{1}(R, \theta) / p_{\mathrm{DC}}$, is given for the same case as Figure 9. The results are similar, but the numerical calculation shows higher current density towards the furnace center than the analytical calculation.

Figure 11 shows the ratio between the numerical and analytical AC resistance factor.* The analytical AC

\footnotetext{
*In Figures 11, 14 and Supplementary Fig. S-4, cubic interpolation (using the SciPy function scipy.interpolate.griddata) has been used to interpolate results from the parameter study. ${ }^{[33]}$
}

resistance factor $R_{\mathrm{AC}, \mathrm{a}}$ is calculated by Eq. [A20]. In other words, Figure 11 shows the correction factor to be
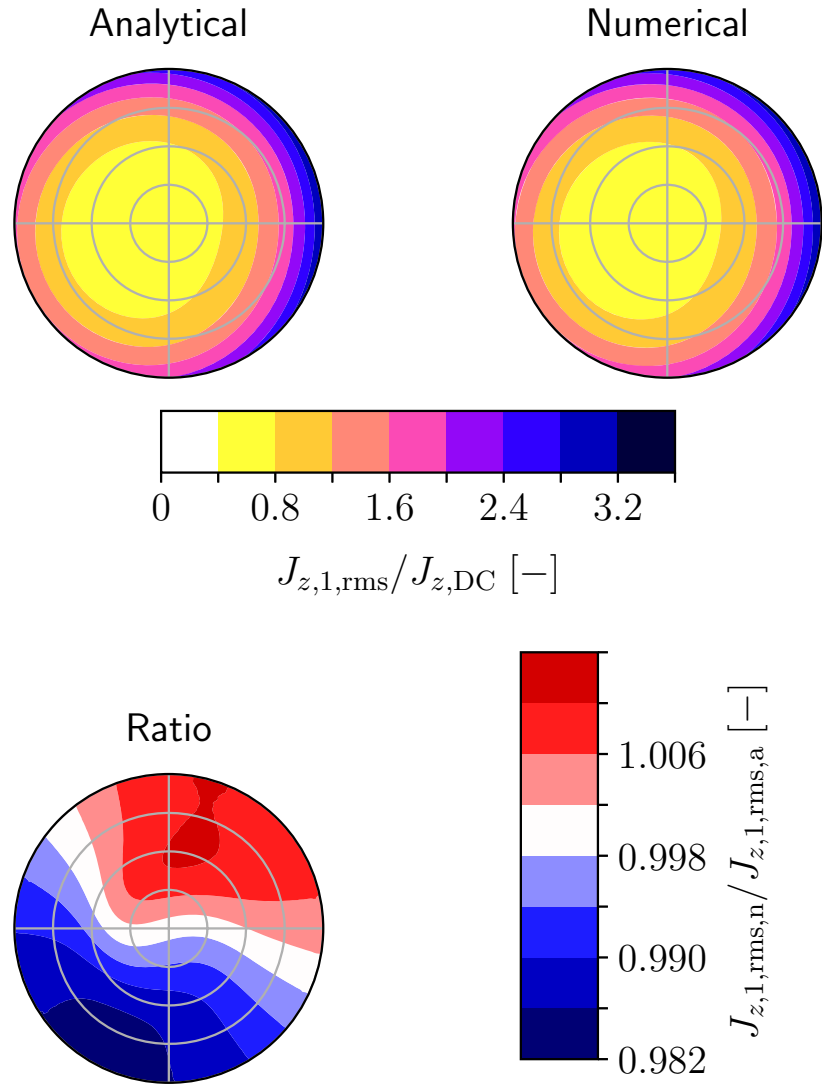

Fig. 9-Contour plots of current density profiles $J_{z, 1, \mathrm{rms}} / J_{z, \text { DC }}$ $(R / \delta=3.0, \quad R / d=0.225)$. Comparison between numerical and analytical results.

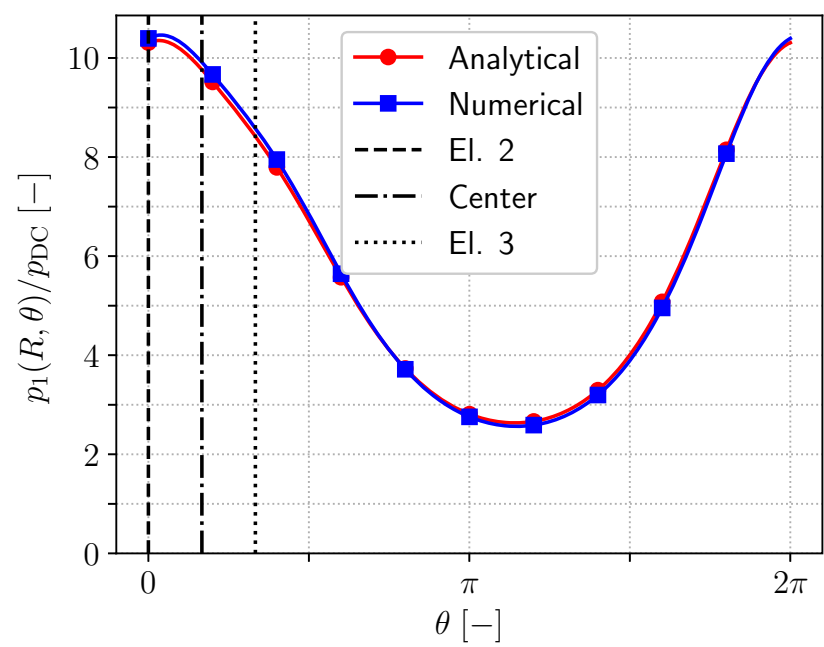

Fig. 10-Normalized power density at the electrode surface $p_{1}(R, \theta) / p_{\text {DC }}$, as a function of $\theta$. Comparison between numerical and analytical results. Directions to other electrodes and furnace center are given by vertical lines (see Fig. 2).

used in Figure 5 and Supplementary Figure S-2, in order to account for distributed currents instead of line currents in the proximate electrodes. The results show that the numerically calculated $R_{\mathrm{AC}}$ values are only marginally different from those calculated analytically. 


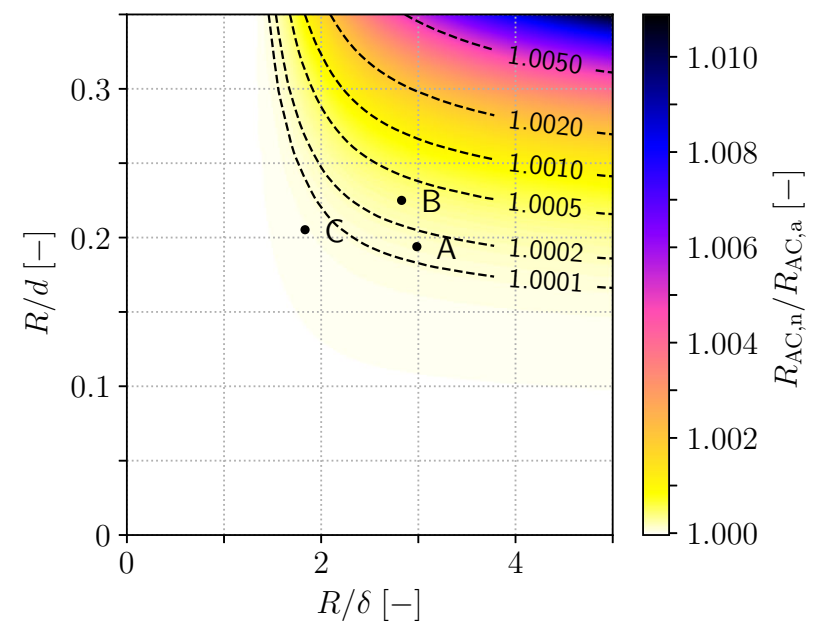

Fig. 11-Ratio between numerically and analytically calculated AC resistance factors as a function of $R / \delta$ and $R / d$. Labels A, B and $\mathrm{C}$ denote the cases of Table I.

The fraction between these values is largest in the upper right corner, when both $R / \delta$ and $R / d$ are large. For the example electrodes of Table I, the fraction is smaller than 1.0004 for all three cases, i.e., the numerically calculated values are $\sim 0.1 \%$ larger for reasonably sized electrodes.

The same comparison between the numerically and analytically calculated values can also be performed for the relative power density variation, and the result is presented in Supplementary Figure S-4. The results show that the difference between the numerically and analytically calculated values are $\sim 1$ pct.

The results presented in this section (Figures 9 to 11) show that deviations are small between the numerical model results and the analytical expressions presented in Section II-B. The differences found can be attributed to the line current approximation of neighboring electrode currents, as assumed in Section II-B. In reality, there is a spatial extent of the electrodes, and thus, currents that are close to other electrodes are more important for proximity effects. Also, because of proximity effects, currents will, on average, be displaced with respect to the electrode center. In most cases, this means that proximity effects are slightly more pronounced than when calculated by the expressions of Section II-B.

\section{B. Triangular Electrode Configuration with Shell}

In this section, results are shown for the general model with a triangular electrode configuration and a shell with finite thickness. This model has been investigated by changing four parameters: $\sigma, \sigma_{\mathrm{sh}}, d$ and $R_{\mathrm{f}}$. These have been varied such that the nondimensional numbers have been studied in intervals $R / \delta \in[0.25,5]$, $R / d \in[0.015,0.45], \quad d /\left(\sqrt{3} R_{\mathrm{f}}\right) \in[0.10,0.90] \quad$ and $t_{\mathrm{sh}} / \delta_{\mathrm{sh}} \in[0.1,30.0]$. By assuming that electrodes should not overlap with each other, nor the furnace shell, there are constraints that must be obeyed, $R / d<0.5$ and $d /\left(\sqrt{3} R_{\mathrm{f}}\right)<(1+\sqrt{3} R / d)^{-1}$.

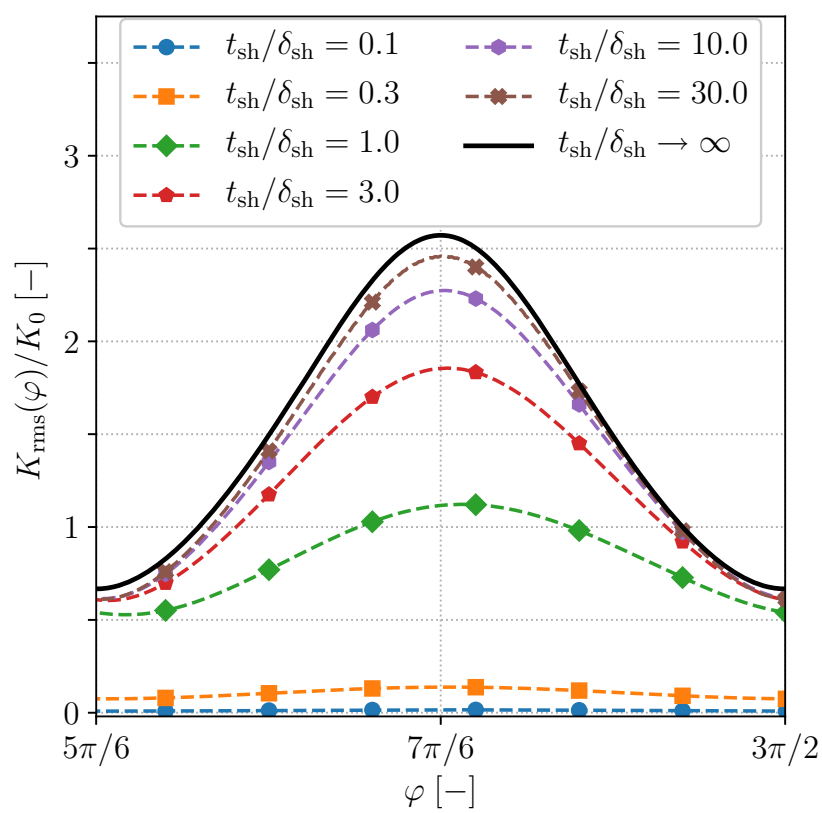

Fig. 12-Normalized shell currents, $K_{\mathrm{rms}}(\varphi) / K_{0}$ as a function of $\varphi$, with $R / \delta=3, R / d=0.25$ and $d /\left(\sqrt{3} R_{\mathrm{f}}\right)=0.5$. Results are for the electrode 1 section (when $\varphi \in[5 \pi / 6,3 \pi / 2$ ), see Fig. 6). Dashed curves show results from numerical simulations, and the solid line is given by the analytical expression (Eq. A21]).

\section{Shell currents}

With the numerical model, it is possible to study how the induced shell currents are affected by the finite thickness of the shell. In Figure 12, results for the normalized shell currents $K_{\mathrm{rms}}(\varphi) / K_{0}$ are shown in the case where $R / \delta=3, R / d=0.25$ and $d /\left(\sqrt{3} R_{\mathrm{f}}\right)=0.5$. In the numerical model, the shell currents are modelled explicitly as volume currents, $J_{z, \mathrm{sh}}(s, \varphi)$, where $s$ is the radial coordinate of the furnace. The corresponding surface currents are then found by integration, $K(\varphi)=$ $\int_{R_{\mathrm{f}}}^{R_{\mathrm{f}}+t_{\mathrm{sh}}} J_{z, \mathrm{sh}}(s, \varphi)$, such that they can be compared with the analytical model of Eq. [A21]. The figure shows that the analytical expression (black curve) is consistent with the numerical model, only if $t_{\mathrm{sh}} / \delta_{\mathrm{sh}} \gg 1$, as should be expected due to the assumptions in its derivation. The deviations from the analytical expression are large when $\delta_{\mathrm{sh}}$ is on the order of or larger than $t_{\mathrm{sh}}$. Also, there are small deviations caused by the finite extent of the electrodes.

For the purpose of studying the strength of the shell currents, results for the shell volume currents can be integrated in sections determined by the nearest electrode, similar to Eq. [A23]. In Figure 13, results are shown in the case when $R / \delta=3$ and $R / d=0.25$, and the strong dependence on both $d /\left(\sqrt{3} R_{\mathrm{f}}\right)$ and $t_{\mathrm{sh}} / \delta_{\mathrm{sh}}$ can be seen. The deviations from the analytical expression are relatively small when $t_{\mathrm{sh}} / \delta_{\mathrm{sh}} \geq 10$. However, the analytical expression only captures the variation that depends on $d /\left(\sqrt{3} R_{\mathrm{f}}\right)$. In addition to the strong dependence on $t_{\mathrm{sh}} / \delta_{\mathrm{sh}}$, the shell current strength also depends on $R / \delta$ and $R / d$, as can be seen in Figure 14. In reality, the electrodes have a finite extent with spatial variations 


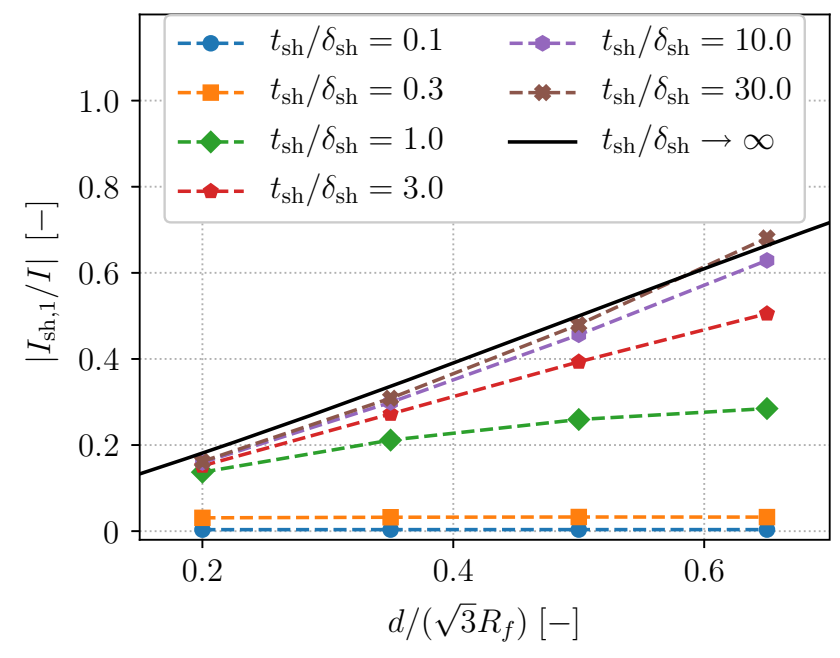

Fig. 13-Shell current, $\left|I_{\mathrm{sh}, 1} / I\right|$ as a function of $d /\left(\sqrt{3} R_{\mathrm{f}}\right)$, with $R / \delta=3$ and $R / d=0.25$. Dashed curves show results from numerical simulations, and the solid line is given by the analytical expression in Eq. [A23].

both for the amplitude and phase of the current density. This will affect the distribution and phase of the induced shell currents. For instance, in cases with strong electrode-electrode proximity effects, the current density will shift towards the leading electrode, and accumulate closer to the furnace center. This will effectively reduce the induced shell currents, since the distance to the shell effectively increases.

\section{Electrode currents}

Similar to Section IV-A, we study the current and power distribution of the electrodes, but now including the interactions with the shell currents.

In Figure 15, results for the normalized current densities are shown for different values of the nondimensional number $d /\left(\sqrt{3} R_{\mathrm{f}}\right)$. In all cases, $R / \delta=3$, $R / d=0.20$ and $t_{\mathrm{sh}} / \delta_{\mathrm{sh}}=10$. When $d /\left(\sqrt{3} R_{\mathrm{f}}\right)$ is small, the shell is distant compared with the electrode-electrode distance, and the current density profile is similar to that found by using the analytical expression in Eq. [A15], which includes electrode-electrode proximity effects, but not proximity effects with the shell currents. However, when $d /\left(\sqrt{3} R_{\mathrm{f}}\right)$ increases, a shift in accumulation of currents from the inside to the outside of the electrode is observed. This accumulation is caused by electrode-shell proximity effects that effectively "compete" with the electrode-electrode proximity effects. The case with $d /\left(\sqrt{3} R_{\mathrm{f}}\right)=0.65$ represents a furnace where the electrodes are very close to the shell, whereas the industrial cases shown in Table I have $d /\left(\sqrt{3} R_{\mathrm{f}}\right) \approx 0.4$. Note that for $d /\left(\sqrt{3} R_{\mathrm{f}}\right)=0.5$, the current density is fairly axisymmetric, not very different from the case with only skin effect, as shown in Figure 3.

The effects of including the steel shell in the model can also be seen by studying numerical results for the AC resistance factor $R_{\mathrm{AC}} / R_{\mathrm{DC}}$ in Figure 16 . Here, three nondimensional numbers $R / \delta, R / d$ and $d /\left(\sqrt{3} R_{\mathrm{f}}\right)$ have

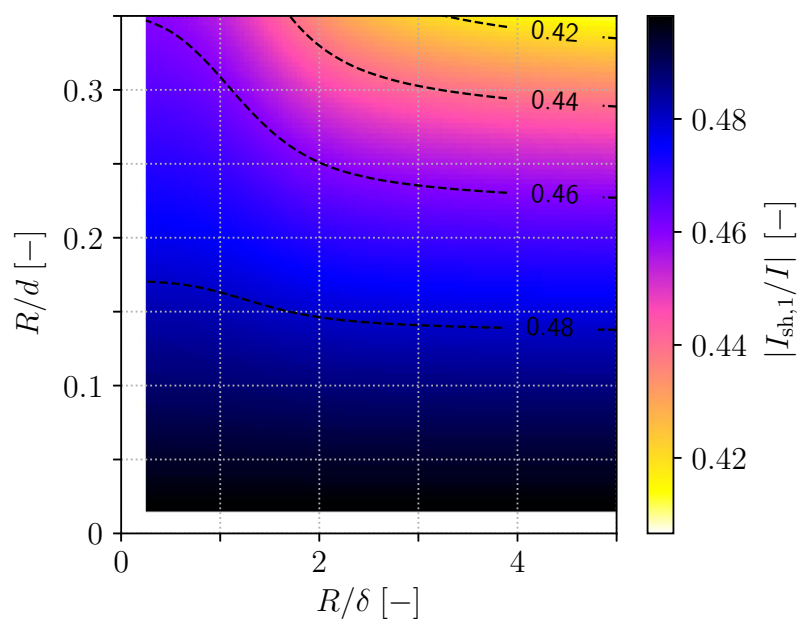

Fig. 14-Shell current, $\left|I_{\mathrm{sh}, 1} / I\right|$ as a function of $R / \delta$ and $R / d$ with $d /\left(\sqrt{3} R_{\mathrm{f}}\right)=0.5$ and $t_{\mathrm{sh}} / \delta_{\mathrm{sh}}=10$. In this case, Eq. [A23] yields $\left|I_{\mathrm{sh}, 1} / I\right|=0.5$. The area with white colors, $(R / \delta<0.25) \cup$ $(R / d<0.015)$, was outside the parameter range for these simulations.
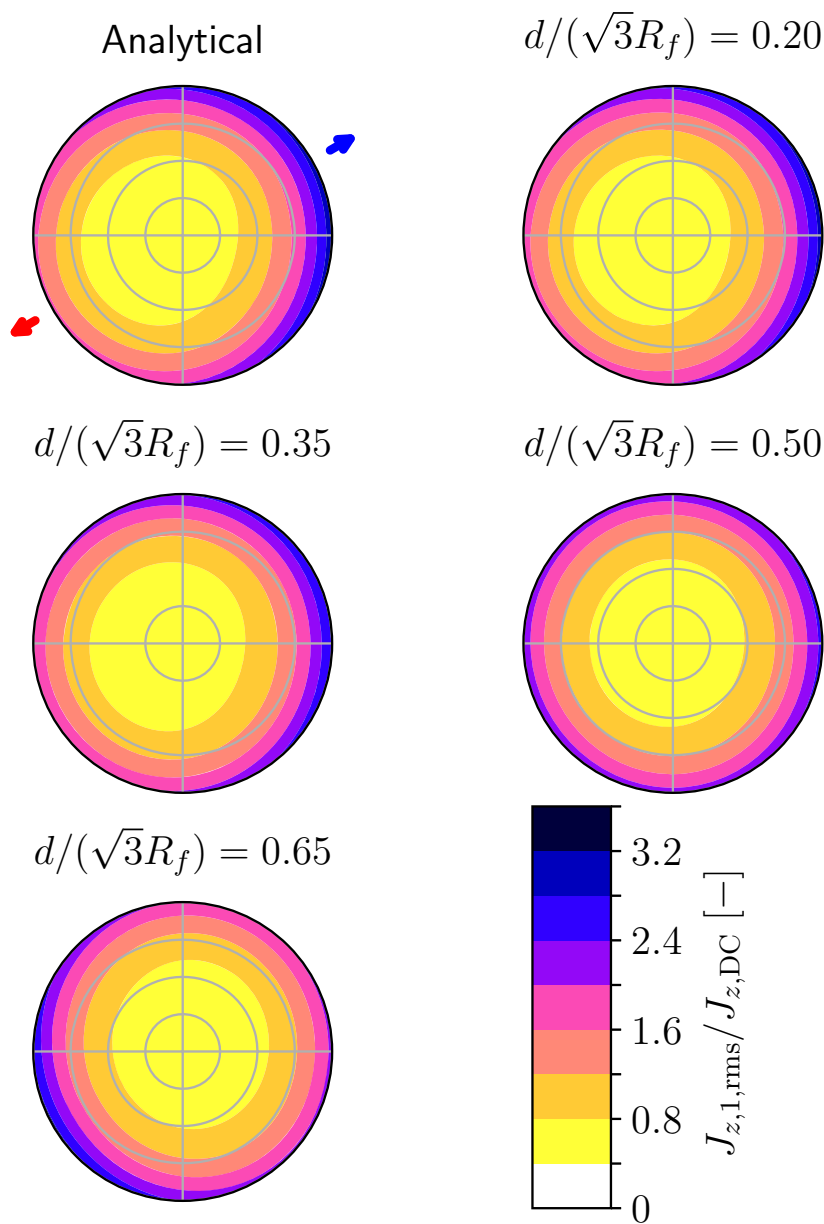

Fig. 15-Contour plots of current density profiles for electrode 1, when $R / \delta=3.0, R / d=0.20$ and $t_{\mathrm{sh}} / \delta_{\mathrm{sh}}=10$. Results are given for four different values of $d /\left(\sqrt{3} R_{\mathrm{f}}\right)$. Blue and red arrows show directions toward the furnace center and steel shell, respectively. 


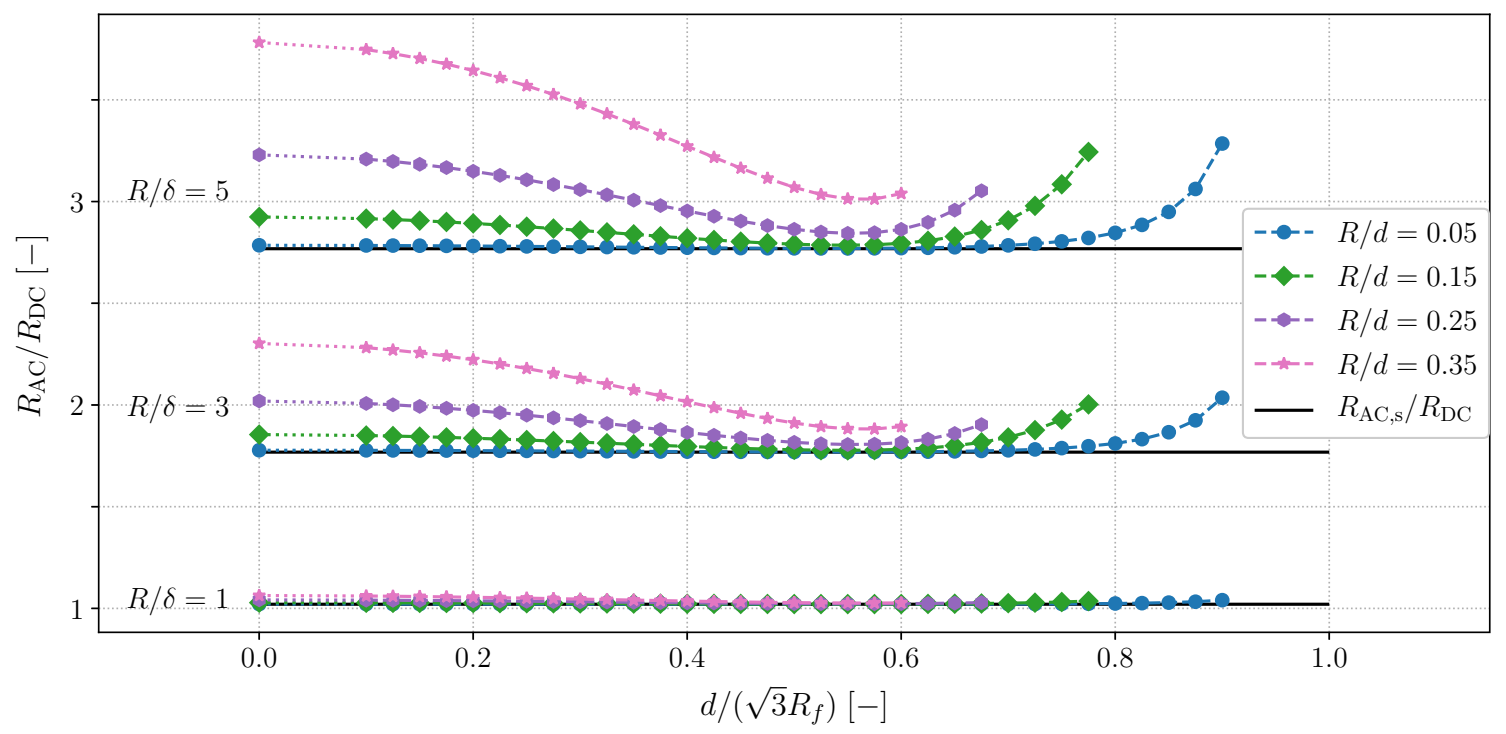

Fig. 16- AC resistance factor $R_{\mathrm{AC}} / R_{\mathrm{DC}}$ as a function of $d /\left(\sqrt{3} R_{\mathrm{f}}\right)$ for different values of $R / d$ and $R / \delta$, and with $t_{\mathrm{sh}} / \delta_{\mathrm{sh}}=10$. Results are calculated using the model including the furnace shell (Section IV-B), except when $d /\left(\sqrt{3} R_{\mathrm{f}}\right)=0$, which are calculated using the model without the furnace shell (Section IV-A). Solid black horizontal lines show the AC skin effect factor (Eq. [A7]).

been varied, whereas the nondimensional shell thickness is constant; $t_{\mathrm{sh}} / \delta_{\mathrm{sh}}=10$. The main features related to $R / \delta$ and $R / d$ can be understood by the simpler models studied in Sections II-A, II-B and IV-A. That is, the $\mathrm{AC}$ resistance factor increases with more skin effects, quantified by the skin effect factor $R_{\mathrm{AC}, \mathrm{s}} / R_{\mathrm{DC}}$ which only depends on $R / \delta$. Moreover, $R_{\mathrm{AC}} / R_{\mathrm{DC}}$ increases with more electrode-electrode proximity effects, quantified by both $R / d$ and $R / \delta$. Note that the results with $d /\left(\sqrt{3} R_{\mathrm{f}}\right)=0$ in Figure 16 were calculated using the model without the furnace shell (Section IV-A), which is approximately equal to Eq. [A20] (see Figure 11).

The most interesting feature of Figure 16 is that the AC resistance factor also depends on furnace radius $R_{\mathrm{f}}$. For all cases of $R / \delta$ and $R / d$, the AC resistance factor decreases monotonically with $d /\left(\sqrt{3} R_{\mathrm{f}}\right)$ until it reaches a minimum at $d /\left(\sqrt{3} R_{\mathrm{f}}\right) \approx 0.55$. Above this value, the $\mathrm{AC}$ resistance factor increases. These results clearly show the competition between the electrode-electrode and the electrode-shell proximity effects. The first dominates for small $d /\left(\sqrt{3} R_{\mathrm{f}}\right)$ values, and the second dominates for large $d /\left(\sqrt{3} R_{\mathrm{f}}\right)$ values. In between, there is an intermediate region where the two proximity effects compete, effectively reducing the asymmetry in the current distribution of the electrode. The same qualitative behavior can also be seen when studying the power density asymmetry at the electrode surface. Figure 17 shows the power density at the electrode surface for different values of $d /\left(\sqrt{3} R_{\mathrm{f}}\right)$ as a function of the azimuthal angle of the electrode. The values of the other nondimensional numbers are $R / \delta=3, R / d=0.2$ and $t_{\mathrm{sh}} / \delta_{\mathrm{sh}}=10$. With increasing values of $d /\left(\sqrt{3} R_{\mathrm{f}}\right)$, the peak towards the leading electrode is reduced, whereas at higher values another peak emerges, directed towards the shell.

\section{Comparison with $3 D$ Results}

The 2D models investigated in this work represent the horizontal cross sections of three-phase AC furnaces with triangular electrode configuration. Models without the furnace shell represent cross sections in regions above the furnace edge, and models with the furnace shell represent cross sections below the furnace edge. However, for the 2D models to be quantitatively accurate, the vertical length of the regions must be large compared with the horizontal extent of the system, and this is not the case for 3D industrial furnaces. Particularly, when it comes to results for induced currents in the steel shell, we expect that the 2D results are quantitatively different compared with $3 \mathrm{D}$. The reason for this is that the induced shell currents are expected to be smaller in $3 \mathrm{D}$, because of the finite height of the steel shell.

In Reference 28, 3D models of furnaces A and B of Table I, were studied. The results for induced shell currents $\left|I_{\mathrm{sh}, 1} / I\right|$ have been compared with the $2 \mathrm{D}$ models presented in this paper, and the results are given in Table III. In addition to the properties given in Table I, $t_{\mathrm{sh}} / \delta_{\mathrm{sh}}=6.08$ and 19.95 have been used for furnace $\mathrm{A}$ and $\mathrm{B}$, respectively. The strength of the induced currents in the steel shell is significantly reduced for the 3D model compared with the 2D models.

Also, Reference 28 shows results for the power density variation on the electrode surface (see Eq. [3]). In Table IV, these results are compared with $2 \mathrm{D}$ model results.** For two of the $3 \mathrm{D}$ cross

\footnotetext{
**For furnace B, only one cross section is compared since the other cross section with $z=1.30 \mathrm{~m}$, is located between the electrode tip and the crater wall. Here, the power density is highly affected by $3 \mathrm{D}$ effects.
} 


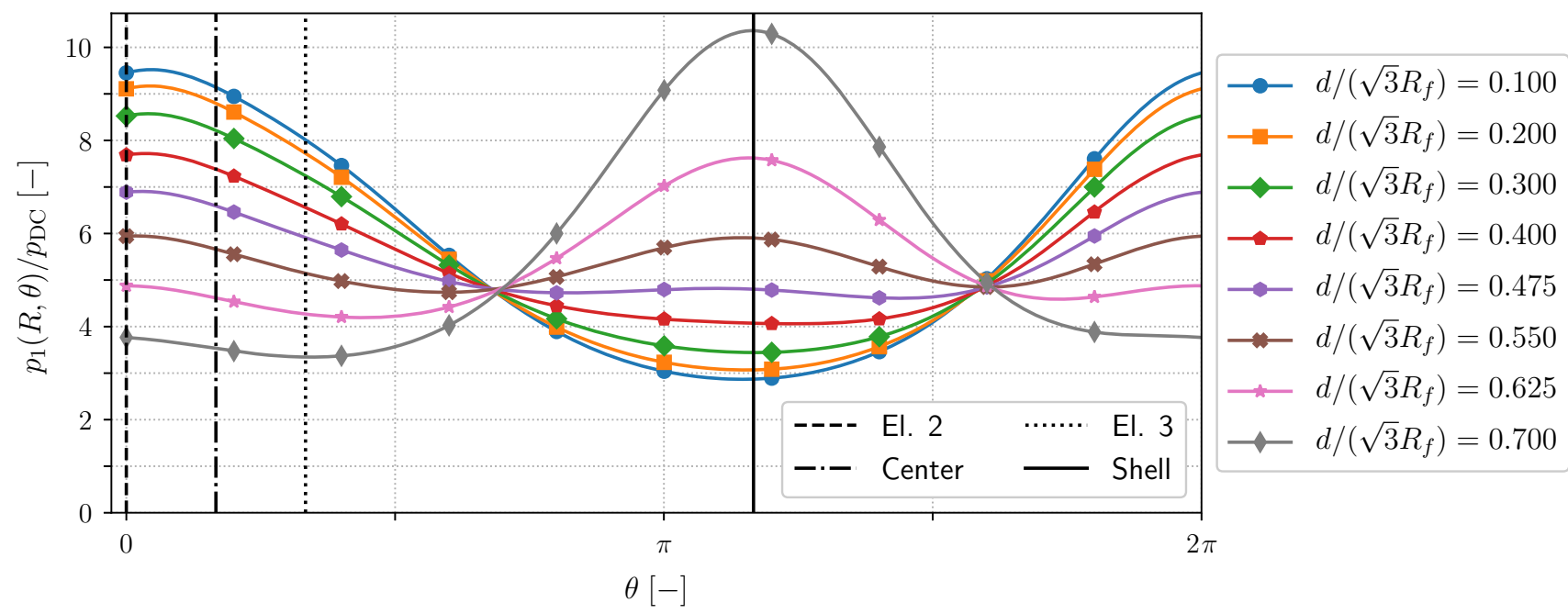

Fig. 17-Normalized power density at the electrode surface $p_{1}(R, \theta) / p_{\mathrm{DC}}$, as a function of $\theta$ with $R / \delta=3, R / d=0.2$ and $t_{\mathrm{sh}} / \delta_{\mathrm{sh}}=10$. Directions to other electrodes, furnace center and furnace shell are given by vertical lines.

Table III. Comparison of Model Results for the Induced Shell Currents $\left|I_{\text {sh }, 1} / I\right|$, for Furnaces A and B of Table I

\begin{tabular}{lcc}
\hline Model & $\left|I_{\text {sh }, 1} / I\right|, \mathrm{A}(-)$ & $\left|I_{\text {sh }, 1} / I\right|, \mathrm{B}(-)$ \\
\hline 2D (Eq. [A23]) & 0.395 & 0.412 \\
2D (Section IV-B) & 0.369 & 0.379 \\
3D* & 0.266 & 0.223 \\
\hline
\end{tabular}

*Ref. 28.

Table IV. Comparison of Model Results of the Power Density Variation $\Delta p_{R} / \overline{p_{R}}$, for Different Electrode Cross Sections of Furnaces A and B of Table I

\begin{tabular}{lccc}
\hline Model & $\begin{array}{c}\Delta p_{R} / \overline{p_{R}}, \\
\mathrm{~A} 1(-)\end{array}$ & $\begin{array}{c}\Delta p_{R} / \overline{p_{R}}, \\
\mathrm{~A} 2(-)\end{array}$ & $\begin{array}{c}\Delta p_{R} / \overline{p_{R}} \\
\text { B1 }(-)\end{array}$ \\
\hline 2D (Eq. [A13]) & 1.13 & 1.13 & 1.31 \\
2D (Section IV-A) & 1.15 & 1.15 & 1.34 \\
2D (Section IV-B) & 0.62 & 0.62 & 0.72 \\
3D* & 1.11 & 0.94 & 1.31 \\
\hline
\end{tabular}

A1 and A2 mean results for the cross sections at $z=5.45 \mathrm{~m}$ and $z=3.25 \mathrm{~m}$ of furnace A, respectively. B1 means results for the cross section at $z=4.15 \mathrm{~m}$ of furnace $\mathrm{B}$.

*Ref. 28

sections ( $\mathrm{A} 1$ and $\mathrm{B} 1$ ), the $3 \mathrm{D}$ results are similar to the 2D models without a steel shell. These cross sections are on the level of or above the furnace edge. For cross section $\mathrm{A} 2$, the $3 \mathrm{D}$ result is in between the $2 \mathrm{D}$ model results with and without the furnace shell. This cross section is below the furnace edge, and hence, the power density variation is reduced by the electrode-shell proximity effect. The effect is less pronounced than what is predicted by the $2 \mathrm{D}$ model, but the values still suggest that electrode-shell proximity effects yield a significant correction to the current and power density of the electrodes. Hence, electrode-shell proximity should be considered for optimal accuracy of electrode models since it effectively will reduce the variations in current density caused by the electrode-electrode proximity effect and reduce the estimates of the electrode load factor. Another consequence of the induced shell currents is that they generate some heat in the furnace shell. Although the amount is small compared with the total furnace power, ${ }^{[28]}$ the heating may affect local temperatures in the lining and shell. ${ }^{[27]}$

\section{CONCLUSIONS}

2D models describing the skin effect, electrode-electrode proximity effects and currents in the furnace shell have been reviewed. The models reveal how these effects depend on furnace and electrode sizes, as well as the location of the electrodes. In a large furnace, there will be considerable skin and proximity effects in the electrodes.

The nondimensional number $R / \delta$ quantifies the strength of induction effects in the electrodes. When this number is small, the current density in the electrode is uniform, and when this number is large, the current flows only close to the periphery. For electrodes in large industrial furnaces, $R / \delta$ is larger than 1 , and there is a considerable accumulation of current close to the surface of the electrode.

For the electrode-electrode proximity effect, the nondimensional number $R / d$, which is the ratio between the electrode radius and the electrode distance, is also important. With increasing value of $R / d$, large proximity effects can be noticed, with higher current densities towards the leading electrode. 
The electrode currents will also induce currents in the furnace shell. These currents can be large, given that the ratio between the thickness and the skin depth of the furnace shell, $t_{\mathrm{sh}} / \delta_{\mathrm{sh}}>1$. Where a magnetic steel shell is used, $t_{\mathrm{sh}} / \delta_{\mathrm{sh}} \gg 1$, and large currents will be induced in a thin layer on the inside of the furnace shell. The strength of these shell currents is determined by the ratio between the "electrode circle radius" and the furnace radius, $d /\left(\sqrt{3} R_{\mathrm{f}}\right)$.

The analytical expressions have been compared with numerical models that account for the finite extents of the electrodes and the furnace shell. When shell currents are neglected, the analytical expressions are accurate. However, the analytical models do not account for the electromagnetic interactions between the shell and the electrodes. The numerical simulations show that strong shell currents will have a significant impact on the current distribution within the electrodes. This is the electrode-shell proximity effect, and it effectively competes with the electrode-electrode proximity effect. In industrial furnaces, both effects need to be considered. The outcome of the competition depends, to a large degree, on the value of $d /\left(\sqrt{3} R_{\mathrm{f}}\right)$.

The 2D numerical simulations have been compared to 3D case studies. For industrial furnaces, the shell height is comparable to its diameter, and the region with vertical shell currents should therefore be relatively small. Hence, the shell currents are not properly approximated by $2 \mathrm{D}$ models. The simulations show that the shell currents are significantly smaller than predicted by the 2D models, but they are sufficiently strong to cause a significant reduction in the electrodeelectrode proximity effects. 3D simulations are therefore recommended to gain proper insight into the current distribution within the electrodes in large industrial furnaces.

\section{ACKNOWLEDGMENTS}

This paper is published as part of the project Electrical Conditions and their Process Interactions in High Temperature Metallurgical Reactors (ElMet), with financial support from The Research Council of Norway and the companies Elkem and Eramet Norway.

\section{OPEN ACCESS}

This article is distributed under the terms of the Creative Commons Attribution 4.0 International License (http://creativecommons.org/licenses/by/4.0/), which permits unrestricted use, distribution, and reproduction in any medium, provided you give appropriate credit to the original author(s) and the source, provide a link to the Creative Commons license, and indicate if changes were made.

\section{APPENDIX A}

Appendix A.1: Low-Frequency Maxwell's Equations

The electromagnetic conditions in the furnace are governed by Maxwell's equations, written

$$
\begin{gathered}
\nabla \cdot \mathbf{E}=\frac{\rho_{\mathrm{f}}}{\varepsilon}, \\
\nabla \cdot \mathbf{B}=0, \\
\nabla \times \mathbf{E}=-\frac{\partial \mathbf{B}}{\partial t}, \\
\nabla \times \mathbf{B}=\mu \mathbf{J}+\mu \varepsilon \frac{\partial \mathbf{E}}{\partial t},
\end{gathered}
$$

where $\rho_{\mathrm{f}}$ is the free charge density and $\varepsilon$ is the electric permittivity. The electric current density is assumed to obey Ohm's law, given in Eq. [1]. The furnaces studied here operate at frequencies $f=50$ or $60 \mathrm{~Hz}$, which means that the electric displacement term $\mu \varepsilon \partial \mathbf{E} / \partial t$ in Eq. [A1d] is small and can be neglected. ${ }^{[34,35]}$ Also, in these furnaces, there is no accumulation of charge, so $\rho_{\mathrm{f}}=0$. All electromagnetic fields $\mathbf{A}$ are assumed to be time-harmonic, and can be written $\mathbf{A}(\mathbf{r}, t)=$ $\operatorname{Re}[\mathbf{A}(\mathbf{r}) \exp (\mathrm{i} \omega t)]$, where $\omega=2 \pi f$. Under these assumptions, Maxwell's equations can be written

$$
\begin{gathered}
\nabla \cdot \mathbf{E}=0, \\
\nabla \cdot \mathbf{B}=0, \\
\nabla \times \mathbf{E}=-\mathrm{i} \omega \mathbf{B}, \\
\nabla \times \mathbf{B}=\mu \mathbf{J},
\end{gathered}
$$

where all fields are written in terms of its complex amplitude field.

By taking the curl of Eq. [A2c], inserting Eqs. [A2d] and [1], and using the vector identity

$$
\nabla \times(\nabla \times \mathbf{E})=\nabla(\nabla \cdot \mathbf{E})-\nabla^{2} \mathbf{E}
$$

together with Eq. [A2a], it is possible to obtain an equation for the electrical field only, written

$$
\nabla^{2} \mathbf{E}=\mathrm{i} \frac{2}{\delta^{2}} \mathbf{E} .
$$

The prefactor of the right-hand side has been written in terms of the skin depth $\delta$. When Eq. [A4] is solved for conductive domains, simple nondimensional analysis shows that the term of the right-hand side cannot be neglected when $\delta$ is on the order of or smaller than 
the characteristic size of the conductor. The solution then exhibits a decay of the electric field inside the surface of the conductor; this is the skin effect.

\section{Appendix A.2: Skin Effect Model Expressions}

Equation [A4] can be solved to find useful expression for the current density in AC electrodes (see Figure 1). In the following, the most important results will be quoted; for a derivation of these expressions, References 11 and 12 can be consulted.

The solution for the current density is

$$
J_{z}(r)=\frac{I(1-\mathrm{i})}{2 \pi R \delta} \frac{\mathrm{J}_{0}[(1-\mathrm{i}) r / \delta]}{\mathrm{J}_{1}[(1-\mathrm{i}) R / \delta]},
$$

where $r=\sqrt{x^{2}+y^{2}}$ is the radial coordinate inside the electrode and where $\mathbf{J}_{v}(x)$ is the $v$ th order Bessel function of the first kind. The impedance associated with a section of length $L$ is

$$
Z=\frac{R_{\mathrm{DC}}(1-\mathrm{i}) R}{2 \delta} \frac{\mathrm{J}_{0}[(1-\mathrm{i}) R / \delta]}{\mathrm{J}_{1}[(1-\mathrm{i}) R / \delta]},
$$

where $R_{\mathrm{DC}}=L /\left(\sigma \pi R^{2}\right)$ is the $\mathrm{DC}$ resistance of that section. The AC resistance $R_{\mathrm{AC}}=\operatorname{Re}(Z)$ and reactance $X=\operatorname{Im}(Z)$ are written

$$
\begin{gathered}
R_{\mathrm{AC}}=\frac{R_{\mathrm{DC}} \xi}{2}\left(\frac{\mathrm{bei}_{0} \xi \mathrm{bei}_{0}{ }^{\prime} \xi-\mathrm{bei}_{0} \xi \mathrm{bei}_{0}{ }^{\prime} \xi}{m_{1}}\right), \\
X=\frac{R_{\mathrm{DC}} \xi}{2}\left(\frac{\text { ber }_{0} \xi \mathrm{ber}_{0}{ }^{\prime} \xi+\mathrm{bei}_{0} \xi \mathrm{bei}_{0}{ }^{\prime} \xi}{m_{1}}\right),
\end{gathered}
$$

where the complex-valued Bessel functions have been written in terms of the real-valued Kelvin functions ber $_{v}$, bei $_{v}$ as follows: ${ }^{[36,37]}$

$$
\mathrm{J}_{v}\left[\frac{(1-\mathrm{i}) r}{\delta}\right]=(-1)^{v}\left[\operatorname{ber}_{v}\left(\frac{\xi r}{R}\right)+\mathrm{ibei}_{v}\left(\frac{\xi r}{R}\right)\right] .
$$

and where first-order Kelvin functions have been written in terms of derivatives of zero-order Kelvin functions. Also,

$$
\xi=\frac{\sqrt{2} R}{\delta}
$$

and

$$
m_{n}=\operatorname{ber}_{n}^{2} \xi+\operatorname{bei}_{n}^{2} \xi .
$$

For current-carrying electrodes in metal-producing furnaces, one is typically interested in the root-meansquared current density, written

$$
J_{z, \mathrm{rms}}(r)=\frac{J_{z, \mathrm{DC}} \xi}{2} \sqrt{\frac{\operatorname{ber}_{0}^{2}(\xi r / R)+\mathrm{bei}_{0}^{2}(\xi r / R)}{m_{1}}} .
$$

Appendix A.3: Skin and Proximity Effect Model Expressions

Expressions for the current density of the electrodes in Figure 2 were calculated by Reference 20 . With balanced electrodes and 120-degree phase difference between the electrodes, the electrode currents are written $I_{1}=I, I_{2}=$ $I \exp (\mathrm{i} 2 \pi / 3)$ and $I_{3}=I \exp (-\mathrm{i} 2 \pi / 3)$. The resulting series expansion for the current density of electrode 1 is

$$
\begin{aligned}
J_{z, 1}(r, \theta)= & \frac{I_{1}(1-\mathrm{i})}{2 \pi R \delta}\left\{\frac{\mathrm{J}_{0}[(1-\mathrm{i}) r / \delta]}{\mathrm{J}_{1}[(1-\mathrm{i}) R / \delta]}\right. \\
& \left.-2 \sum_{n=1}^{\infty}\left(\frac{R}{d}\right)^{n} \frac{\mathrm{J}_{n}[(1-\mathrm{i}) r / \delta]}{\mathrm{J}_{n-1}[(1-\mathrm{i}) R / \delta]} f_{n}(\theta)\right\},
\end{aligned}
$$

where

$$
\begin{aligned}
f_{n}(\theta)= & f_{n, r}(\theta)+\mathrm{i} f_{n, c}(\theta) \\
= & \cos \left[n\left(\theta-\frac{\pi}{6}\right)\right] \cos \left[\frac{n \pi}{6}\right] \\
& +\mathrm{i} \sqrt{3} \sin \left[n\left(\theta-\frac{\pi}{6}\right)\right] \sin \left[\frac{n \pi}{6}\right] .
\end{aligned}
$$

The expression for the root-mean-squared current density is

$$
J_{z, 1, \mathrm{rms}}(r, \theta)=\frac{J_{z, \mathrm{DC}} \xi}{2} \sqrt{g^{2}(r, \theta)+h^{2}(r, \theta)},
$$

where

$$
\begin{aligned}
g(r, \theta)= & \frac{s_{0,1}(r)}{m_{1}} \\
& -2 \sum_{n=1}^{\infty}\left(\frac{R}{d}\right)^{n} \frac{s_{n, n-1}(r) f_{n, r}(\theta)-p_{n, n-1}(r) f_{n, c}(\theta)}{m_{n-1}}
\end{aligned}
$$

$$
\begin{aligned}
h(r, \theta)= & \frac{p_{0,1}(r)}{m_{1}} \\
& -2 \sum_{n=1}^{\infty}\left(\frac{R}{d}\right)^{n} \frac{s_{n, n-1}(r) f_{n, c}(\theta)+p_{n, n-1}(r) f_{n, r}(\theta)}{m_{n-1}}
\end{aligned}
$$

$$
s_{m, n}(r)=\operatorname{ber}_{m}\left(\frac{\xi r}{R}\right) \operatorname{ber}_{n} \xi+\operatorname{bei}_{m}\left(\frac{\xi r}{R}\right) \operatorname{bei}_{n} \xi,
$$

and

$$
p_{m, n}(r)=\operatorname{bei}_{m}\left(\frac{\xi r}{R}\right) \operatorname{ber}_{n} \xi-\operatorname{ber}_{m}\left(\frac{\xi r}{R}\right) \operatorname{bei}_{n} \xi .
$$

The resistance is given by 


$$
\begin{aligned}
R_{\mathrm{AC}}= & \frac{R_{\mathrm{DC}} \xi}{2}\left\{\frac{\text { ber }_{0} \xi \mathrm{bei}_{0}^{\prime} \xi-\text { bei }_{0} \xi \text { ber }_{0}^{\prime} \xi}{m_{1}}\right. \\
& +2 \sum_{n=1}^{\infty}\left(\frac{R}{d}\right)^{2 n}\left[1+2 \sin ^{2}\left(\frac{n \pi}{6}\right)\right] \\
& \left.\times \frac{\operatorname{ber}_{n} \xi \operatorname{bei}_{n}^{\prime} \xi-\text { bei }_{n} \xi \text { ber }_{n}^{\prime} \xi}{m_{n-1}}\right\} .
\end{aligned}
$$

Equivalent expressions can be found for the two other electrodes. ${ }^{[20]}$

\section{Appendix A.4: Shell Current Model Expressions}

The expression for the surface current $K(\varphi)$ in the steel shell of Figure 6 can be found by integrating out the radial dependence in the shell current density expression derived by Reference 30, and then taking the limit $R_{\mathrm{f}} / \delta_{\mathrm{sh}} \rightarrow \infty$ to find

$$
K(\varphi)=-\frac{I}{\pi R_{\mathrm{f}}} \sum_{n=1}^{\infty}\left(\frac{d}{\sqrt{3} R_{\mathrm{f}}}\right)^{n} l_{n}(\varphi),
$$

where

$$
\begin{aligned}
l_{n}(\varphi)= & \cos \left[n\left(\varphi+\frac{5 \pi}{6}\right)\right]+\exp \left(\mathrm{i} \frac{2 \pi}{3}\right) \cos \left[n\left(\varphi+\frac{\pi}{6}\right)\right] \\
& +\exp \left(-\mathrm{i} \frac{2 \pi}{3}\right) \cos \left[n\left(\varphi-\frac{\pi}{2}\right)\right] .
\end{aligned}
$$

It is also instructive to calculate the total induced currents in sections of the shell. When the shell currents are integrated for the full circumference, the sum is zero since currents with opposite phase cancel out. However, by dividing the shell into three sections determined by the nearest electrode, the strength of the shell currents can be studied. For electrode 1, the result is

$$
I_{\mathrm{sh}, 1}=\int_{5 \pi / 6}^{3 \pi / 2} \mathrm{~d} \varphi R_{\mathrm{f}} K(\varphi)=-\frac{3 I}{\pi} \sum_{n=1}^{\infty}\left(\frac{d}{\sqrt{3} R_{\mathrm{f}}}\right)^{n} \frac{\sin (n \pi / 3)}{n} .
$$

\section{ELECTRONIC SUPPLEMENTARY MATERIAL}

The online version of this article (https://doi.org/10.10 07/s11663-019-01651-8) contains supplementary material, which is available to authorized users.

\section{REFERENCES}

1. J. Grzella, P. Sturm, J. Krüger, M. A. Reuter, C. Kögler, and T. Probst: Metallurgical Furnaces, vol. 22, Wiley-VCH Verlag GmbH \& Co. KGaA, Weinheim, 2012, pp. 693-733, https://doi.org/10. 1002/14356007.b04 339.

2. S. Lupi: Fundamentals of Electroheat, 1st ed., Springer International Publishing, Switzerland, 2017.
3. R. Innvær: UIE, Electrotech 92, Montreal, Canada, 1992, pp. 44 53.

4. G. Andersen, R. Innvær, and B. Larsen: INFACON 9, Quebec City, Canada, 2001, pp. 494-500.

5. J. Westly: Electric Furnace Conference, 1984, pp. 157-60.

6. C.R. Ray, P.K. Sahoo, and S.S. Rao: INFACON 11, New Delhi, India, 2007, pp. 741-51.

7. B. Larsen, H. Feldborg, and S.A. Halvorsen: INFACON 13, Almaty, Kazakhstan, 2013, pp. 453-66.

8. J. Westly: Electric Furnace Conference, 1975, pp. 47-53.

9. N.A. Barcza, I.J. Barker, M.S. Rennie, and P.J. Brereton-Stiles: Electric Furnace Conference, 2002, pp. 425-37.

10. M.W. Kennedy: International Smelting Technology Symposium, 2012, vol. 1, pp. 279-90, https://doi.org/10.1002/9781118364765.c h32.

11. S. Ramo, J.R. Whinnery, and T. Van Duzer: Fields and Waves in Communication Electronics, 3rd ed., Wiley, New York, 1994.

12. G.S. Smith: Eur. J. Phys., 2014, vol. 35, p. 025002. http://stacks. iop.org $/ 0143-0807 / 35 / \mathrm{i}=2 / \mathrm{a}=025002$.

13. A. Bermúdez, M.C. Muñiz, F. Pena, and J. Bullón: Int. J.Numer. Methods Eng., 1999, vol. 46, pp. 649-58. https://doi.org/10.1002/ (SICI) 1097-0207(19991020)46:5<649::AID-NME664>3.0.CO;2-C.

14. H.L. Larsen: Proceedings of the 2006 Nordic COMSOL Conference, Copenhagen, Denmark, 2006.

15. I. McDougall: INFACON 11, New Delhi, India, 2007, pp. 63037.

16. Y.A. Tesfahunegn, T. Magnusson, M. Tangstad, and G. Saevarsdottir: 2018 IEEE MTT-S International Conference on Numerical Electromagnetic and Multiphysics Modeling and Optimization (NEMO), Reykjavik, Iceland, 2018, pp. 166-69.

17. Y.A. Tesfahunegn, G. Saevarsdottir, T. Magnusson, and M. Tangstad: 2018 IEEE MTT-S International Conference on Numerical Electromagnetic and Multiphysics Modeling and Optimization (NEMO), Reykjavik, Iceland, 2018, pp. 305-08.

18. Y.A. Tesfahunegn, T. Magnusson, M. Tangstad, and G. Saevarsdottir: 10th International Symposium, ISICA 2018, Jiujiang, China, 2019, pp. 3-14.

19. H.B. Dwight: Trans. Am. Inst. Electr. Eng. (AIEE), 1923, vol. XLII, pp. 850-59.

20. V. Dunski: Elektrowärme, 1962, vol. 20, pp. 503-11.

21. V. Dunski: Elektrowärme, 1962, vol. 20, pp. 616-25.

22. O. Böchmann and L. Olsen: VIth International Congress on Electro-Heat, Brighton, UK, 1968, p. 127.

23. G. Orth: Elektrowärme Int., 1976, vol. 34, pp. 25-30.

24. M.T. Jonsson and H.T. Ingason: INFACON 8, Beijing, China, 1998, pp. 383-88.

25. A. Bermúdez, J. Bullón, and F. Pena: Commun. Numer. Methods Eng., 1998, vol. 14, pp. 581-93. https://doi.org/10.1002/(SIC I) 1099-0887(199806)14:6<581::AID-CNM175>3.0.CO;2-S

26. I. McDougall, C.F.R. Smith, B. Olmstead, and W.A. Gericke: INFACON 10, Cape Town, South Africa, 2004, pp. 575-84.

27. P.F. Ryff: IEEE Trans. Ind. Appl., 1981, vol. IA-17, pp. 329-33.

28. E. Herland, M. Sparta, and S. Halvorsen: J. S. Afr. Inst. Min. Metall., 2018, vol. 118, pp. 607-18.

29. J.A. Tegopoulos and E.E. Kriezis: IEEE Trans. Power Appl. Syst., 1971, vol. PAS-90, pp. 1278-86.

30. K. Kawasaki, M. Inami, and T. Ishikawa: IEEE Trans. Power Appl. Syst., 1981, vol. PAS-100, pp. 474-84.

31. COMSOL Inc.: COMSOL Multiphysics Reference Manual, ver. 5.3, https://www.comsol.com. Accessed 27 December 2018.

32. COMSOL Inc.: AC/DC Module User's Guide, COMSOL Multiphysics 5.3 https://www.comsol.com. Accessed 27 December 2018.

33. SciPy: scipy.interpolate.griddata, https://docs.scipy.org/doc/scipy/ reference/generated/scipy.interpolate.griddata.html. Accessed 27 December 2018.

34. A. Alonso Rodriquez and A. Valli: Eddy Current Approximation of Maxwell Equations, Springer, Milan, 2010.

35. A. Bermúdez, D. Gómez, and P. Salgado: Mathematical Models and Numerical Simulation in Electromagnetism, Springer International Publishing, Cham, 2014.

36. M. Abramowitz and, I.A. Stegun: Handbook of Mathematical Functions with Formulas, Graphs, and Mathematical Tables, 10th ed., National Bureau of Standards, Washington D.C., 1972. http:// people.math.sfu.ca/ cbm/aands/intro.htm. 
37. National Institute of Standards and Technology (NIST): NIST

Digital Library of Mathematical Functions, http://dlmf.nist.gov. Accessed 26 November 2016.
Publisher's Note Springer Nature remains neutral with regard to jurisdictional claims in published maps and institutional affiliations. 